\title{
Electronic and Photovoltaic Properties of $p$-Si/PCBM:MEH-PPV Organic-Inorganic Hybrid Photodiode
}

\author{
F. YAKUPhanOGLU ${ }^{a, b}$ AND W.A. FAROOQ ${ }^{b}$ \\ ${ }^{a}$ Physics Department, Faculty of Science, Firat University, Elazig, Turkey \\ ${ }^{b}$ Department of Physics and Astronomy, College of Science, King Saud University \\ Riyadh, Saudi Arabia
}

(Received August 10, 2010; in final form January 25, 2011)

\begin{abstract}
The photovoltaic and electronic properties of $p$-Si/poly[2-methoxy,5-(2-ethyl-hexyloxy)-1,4-phenylenevinylene] (MEH-PPV):[6, 6]-phenyl $\mathrm{C}_{61}$-butyric acid methyl ester (PCBM) organic-inorganic device have been investigated. The current-voltage characteristic of $p$-Si/PCBM:MEH-PPV photodiode includes series resistance effect and the diode indicates a non-ideal behavior. The photovoltaic effect in $p$-Si/PCBM:MEH-PPV photodiode is based on the formation of excitons and subsequent dissociation and charge collection at the electrodes. It is evaluated that $p$-Si/PCBM:MEH-PPV device is a photodiode with $V_{\mathrm{oc}}$ of $84 \mathrm{mV}$ and $I_{\mathrm{sc}}$ of $3.47 \mathrm{nA}$ electronic parameters.
\end{abstract}

PACS: 85.30.Kk, 88.40.jr

\section{Introduction}

Organic semiconductors are required for electronic and photoelectronic devices due to their functional electrical and optical properties. These materials can be used to fabricate organic-inorganic heterojunctions and organic optoelectronic devices $[1,2]$. An alternative approach for organic-inorganic heterojunctions is to use solution processable organic semiconductors. Organic optoelectronic devices have been object of intense research in recent years, based on advantages as the possibility of producing large areas devices and the possibility to change the optoelectronic features of these materials without considerable changes in the process production [3-10]. Photodiodes based on semiconductor-semiconductor and metal-semiconductor junctions can be fabricated by using different organic semiconductors. When these junctions with organic semiconductor are illuminated, electrons and holes are produced and in turn, these devices show a photovoltaic effect. Organic photovoltaic devices are promising candidates for renewable sources of electrical energy because of ease in fabrication and low production cost as well as light weight and flexibility [11-14]. Photodiodes based on polythiophene and its derivates organic semiconductors and different organic-inorganic heterojunctions such as pyronine- $\mathrm{B} / \mathrm{Si}, \beta$-carotene $/ \mathrm{Si}$, chitin $/ n$-Si and poly[2-methoxy, 5-(2'-ethyl-hexyloxy)-1,4-phenylenevinylene] (MEH-PPV) $/ p$-Si, 4-tricyanovinyl-N,N-diethylaniline $/ p-\mathrm{Si}, \quad \mathrm{Al} / p$-Si/copper phthalocyanine, chitosan $/ n-\mathrm{Si}$, organic/inorganic Schottky,
FSS $/ n$-Si, NiPc $/ p$-Si, Zn(Phen)q $/ p$-Si and ddq $/ p$-Si have been fabricated and their electronic properties have been investigated [15-24]. The prospect of being able to generate electricity from sunlight has fuelled intensive research in inorganic and more recently in organic photovoltaics [25]. [6,6]-phenyl $\mathrm{C}_{61}$-butyric acid methyl ester (PCBM) and MEH-PPV organic semiconductors have been used extensively in optoelectronic devices and solar cells [26, 27]. Polymer photodiodes have been fabricated by combining polymers with fullerene or its soluble derivatives $[28,29]$.

To our best knowledge, the photovoltaic and electronic properties of $p$-Si/PCBM:MEH-PPV device have not been investigated. Therefore, the aim of this study is to fabricate a photodiode based on organic-inorganic heterojunction.

\section{Experimental}

Poly [2-methoxy-5-(2'-ethyl)hexoxy-1,4-phenylenevinylene] and [6,6]-phenyl $\mathrm{C}_{61}$-butyric acid methyl ester organic compounds were purchased from Sigma-Aldrich Co. The chemical structures of the organic compounds are shown in Fig. 1a,b. The blend of organic compounds was prepared as MEH-PPV (20 wt\%) and PCBM (80 wt $\%$ ). $p$-Si [100] wafer was used as $p$-type inorganic semiconductor. In order to remove the native oxide on surface on $p$-Si, the wafer was etched by HF and then was rinsed in deionised water using an ultrasonic bath for 10-15 min and finally was chemically cleaned according 
to method based on successive baths of methanol and acetone. The solution of the blend was homogenized for $1.5 \mathrm{~h}$ and was mixed by ultrasonic and mechanic effects. The thin film of MEH-PPV:PCBM (1:4) blend was prepared by deposition of blend solution on the $p$-Si wafer by dip coating technique [18, 23, 24]. Au finger contact was deposited on MEH-PPV:PCBM $/ p$-Si device by vacuum thermal evaporation. Current-voltage $(I-V)$ characteristics of the device under dark and illumination conditions were measured using a KEITHLEY 2400 sourcemeter. Capacitance-voltage measurement of the diode was performed by a HIOKI 3532-50 LCR. The applied voltage was scanned between -2.0 and $+2.0 \mathrm{~V}$. Photovoltaic measurements were employed using a $200 \mathrm{~W}$ halogen lamp [18].

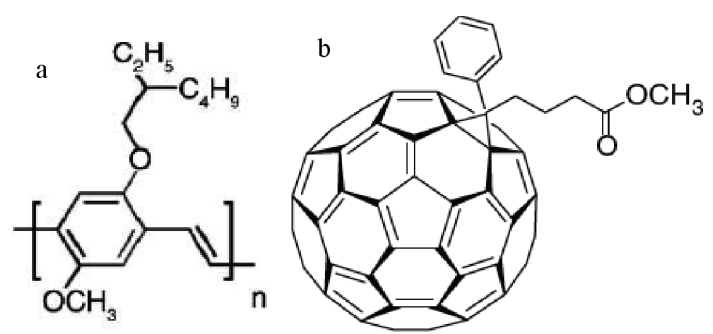

Fig. 1. The chemical structures of the organic compounds (a) MEH-PPV, (b) PCBM.

\section{Results and discussion}

\subsection{Current-voltage characteristics of $p$-Si/PCBM:MEH-PPV diode}

The current-voltage $(I-V)$ characteristics of the $p$-Si PCBM:MEH-PPV device under dark and illumination conditions are shown in Fig. 2. The current under illumination is higher than that of dark current. The illumination increases strongly reverse current of the diode. This suggests that the diode exhibits a photovoltaic behaviour. Figure 2 shows that the diode includes parasitic resistance effects. These effects vary over the $I-V$ range of the diode and series resistance contributes to diode behavior. Under forward bias for $V_{\mathrm{d}}=V-I R_{\mathrm{s}} \gg k T$, the current-voltage expression of a non-ideal heterojunction diode is expressed as [30, 31]:

$$
I=I_{0} \exp \left(\frac{q\left(V-I R_{\mathrm{s}}\right)}{n k T}\right),
$$

where $n$ is the ideality factor, $I_{0}$ is the reverse saturation current, $R_{\mathrm{s}}$ is the series resistance and $q$ is the electronic charge. At higher voltages, series resistance becomes significant on device behavior. In order to determine the diode parameters, we have used the following relations [32]:

$$
\begin{aligned}
& \frac{\mathrm{d} V}{\mathrm{~d} \ln (I)}=n \frac{k T}{q}+I R_{\mathrm{s}}, \\
& H(I)=V-n \frac{k T}{q} \ln \left(\frac{I_{0}}{A A^{*} T^{2}}\right)
\end{aligned}
$$

and

$$
H(I)=I R_{\mathrm{s}}+n \phi_{\mathrm{B}} .
$$

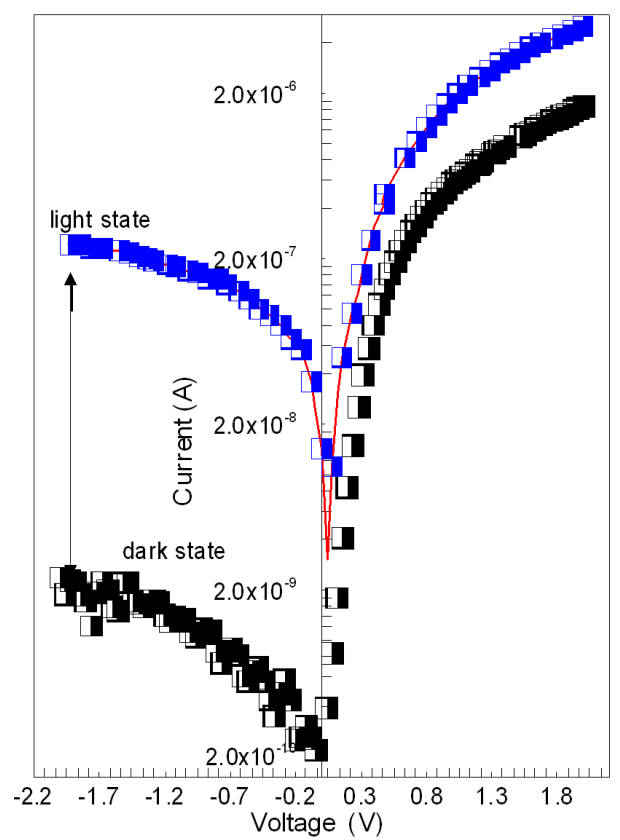

Fig. 2. Current-voltage characteristics of the $p$-Si/ PCBM:MEH-PPV diode at dark and illumination conditions.

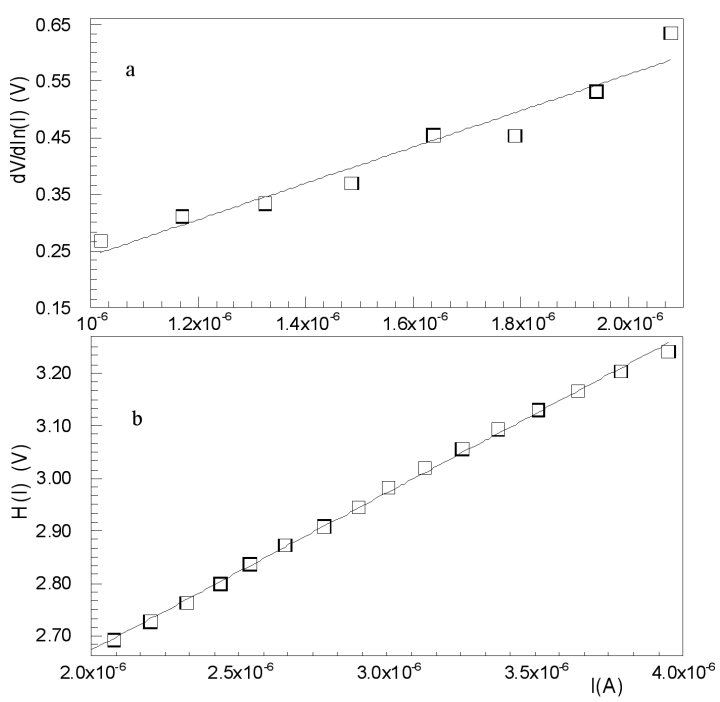

Fig. 3. Plots of $\mathrm{d} V / \mathrm{d} \ln (I)-I$ and $H(I)-I$ of the $p$-Si / PCBM:MEH-PPV diode.

Plots of $\mathrm{d} V / \mathrm{d} \ln I-I$ and $H(I)-I$ are determined from data of the downwards curvature region in the forward 
$I-V$ plot under dark condition and are shown in Fig. 3a,b. The series resistance $R_{\mathrm{s}}$ and ideality factor $n$ values were calculated from Fig. 3a and were found to be $3.20 \times 10^{5} \Omega$ and 3.09 , respectively. The $\phi_{\mathrm{B}}$ and $R_{\mathrm{s}}$ values were determined from the $H(I)-I$ plot and were found to be 0.66 and $3.04 \times 10^{5} \Omega$, respectively. When the voltage is applied to $p$-Si/PCBM:MEH-PPV, a drop in applied voltage can take place due to the interfacial layer and therefore, the barrier height is dependent on the applied voltage, leading to the ideality factor higher than unity. At higher currents, a deviation in the ideality factor results from the interface state density and series resistance. The PCBM:MEH-PPV blend is a semiconductor with low mobility. In this semiconductor, the injection rate is faster than the current flow into the semiconductor, and consequently the injection current is the product of the number of injected carriers. In this case, diffusion-limited thermionic emission model is appropriate and this model is expressed as [33]:

$$
I=q N_{\mathrm{v}} \mu(T) F \exp \left(-\frac{\phi_{\mathrm{b}}-\Delta \phi}{k T}\right),
$$

where $q$ is the electronic charge, $k$ is the Boltzmann constant, $T$ is the temperature, $N_{\mathrm{v}}$ is the effective density of states in the semiconductor, $\phi_{\mathrm{b}}$ is the barrier height, $F$ is the electric field and $\Delta \phi$ is the lowering of the barrier. Figure 4 shows plot of $\ln I$ vs. $1000 / T$ for the device at constant voltage of $0.15 \mathrm{~V}$. Figure 4 indicates a linearity and this linearity is precise evidence that in $p$-Si/ PCBM:MEH-PPV device, the charge carrier transport is limited by thermionic injection. The value of $\phi_{\mathrm{b}}-\Delta \phi$ was determined from the slope of Fig. 4 and was found to be $0.57 \mathrm{eV}$. In diffusion limited injection, the barrier height $\phi_{\mathrm{b}}$ has an important effect in the injection limited. The barrier lowering value for the diode was found to be $0.1 \mathrm{eV}$. At higher voltages, $I-V$ characteristics of the $p$-Si/PCBM:MEH-PPV diode are affected by the transport properties of the organic semiconductor [34]. In order to analyze this effect, $I-V$ curves were plotted in the form of $\log I-\log V$ and are shown in Fig. 5. The $I-V$ curves indicate different current regions. Ohmic region was not shown on these curves. In I, II and III regions, the current changes with $V^{m}$. In $\log I-\log V$ curves, first region suggests a trap controlled space charge limited. The second region indicates that the current is trap filling mechanism. At region III, the increase rate of current with voltage decreases. This suggests that most of traps are filled and contribution of free carrier to electric field becomes appreciable [35].

\subsection{The capacitance-voltage characteristic of $p$-Si/PCBM:MEH-PPV diode}

Figure 6 shows curve of $C^{-2}-V$ of $p$-Si/PCBM:MEH-PPV diode at $1 \mathrm{kHz}$. For abrupt junction, the capacitance of $p-n$ junction is expressed by the following relation [36]:

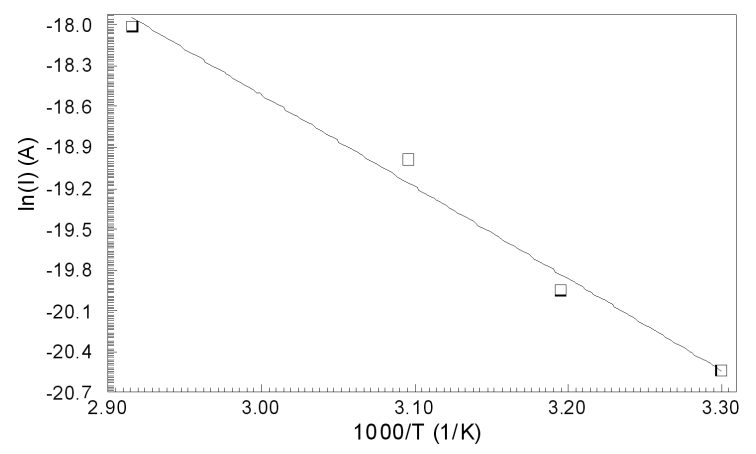

Fig. 4. Plot of $\ln I-1000 / T$ of the $p$-Si/PCBM:MEH-PPV diode.

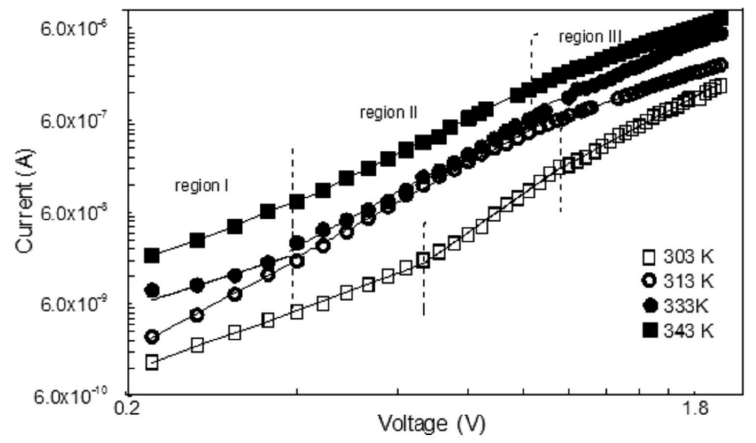

Fig. 5. Plots of $\log I-\log V$ of the $p$-Si/PCBM:MEH-PPV diode at different temperatures.

$$
C^{2}=\frac{q N_{\mathrm{d}} N_{\mathrm{a}} \varepsilon_{1} \varepsilon_{2}}{2\left(\varepsilon_{1} N_{\mathrm{d}}+\varepsilon_{2} N_{\mathrm{a}}\right)} \frac{1}{V_{\mathrm{bi}}-V},
$$

where $V_{\mathrm{bi}}$ is the built-in potential, $\varepsilon_{1}$ is the dielectric constant of $p-\mathrm{Si}, \varepsilon_{2}$ is the dielectric constant of PCBM:MEH-PPV organic semiconductor, $N_{\mathrm{d}}$ and $N_{\mathrm{a}}$ are the donor and acceptor concentrations, respectively. For the $p$-Si/ PCBM:MEH-PPV diode, the depletion layer capacitance is expressed as

$$
N_{\mathrm{a}}=\left(\frac{2}{q \varepsilon_{1} A^{2}}\right) \frac{\mathrm{d} V}{\mathrm{~d} C^{-2}} .
$$

The values of $V_{\mathrm{bi}}$ and $N_{\mathrm{a}}$ can be obtained from the intercept and slope of $C^{-2}$ vs. $V$ plot, respectively. The doping concentration $N_{\mathrm{a}}$ and the built-in potential $V_{\mathrm{bi}}$ values were found to be $5.41 \times 10^{14} \mathrm{~cm}^{-3}$ and $0.83 \mathrm{~V}$, respectively. The barrier height for $p$-Si/PCBM:MEH-PPV diode can be determined using the following relation $[30,36]$ :

$$
\phi_{\mathrm{b}}=V_{\mathrm{bi}}+\frac{k T}{q} \ln \left(\frac{N_{\mathrm{v}}}{N_{\mathrm{a}}}\right),
$$

where $N_{\mathrm{v}}$ value for $\mathrm{Si}$ is $1.82 \times 10^{19} \mathrm{~cm}^{-3}$ [36]. The $\phi_{\mathrm{B}}$ value was determined by means of Eq. (8) and was found to be $1.09 \mathrm{eV}$. The barrier heights deduced from $I-V$ measurement is lower than that of $C-V$ technique. The discrepancy between $\phi_{\mathrm{b}}(C-V)$ and $\phi_{\mathrm{b}}(I-V)$ can be 
explained by existence of excess capacitance and interface properties. The direct current across the interface is exponentially dependent on barrier height and the current is sensitive to barrier distribution at the interface. Whereas the capacitance is insensitive to potential fluctuations on a length scale of less than the space charge width. Consequently, the $\phi_{\mathrm{b}}$ value obtained from $C-V$ measurement is higher than that of $\phi_{\mathrm{b}}$ value obtained from $I-V$ measurements [37].

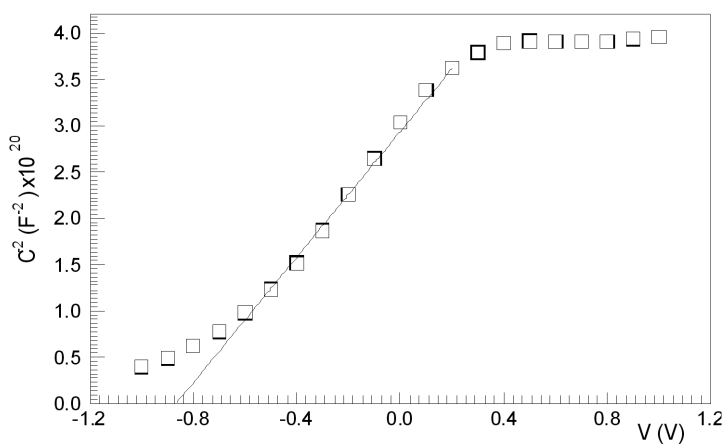

Fig. 6. Plot of $C^{-2}-V$ of $p$-Si/PCBM:MEH-PPV diode.

\subsection{Photovoltaic properties of $p$-Si/ PCBM:MEH-PPV diode}

The current-voltage characteristics of the $p$-Si/ PCBM:MEH-PPV diode under different illumination intensities is shown in Fig. 7. As seen in the figure, the light on reverse $I-V$ characteristic is translated downward and moved in the $-I$ direction along the current axis, giving an open circuit voltage $\left(V_{\text {oc }}\right)$ along with a short photocurrent $\left(I_{\mathrm{Sc}}\right)$. The current in reverse direction is strongly increased by illumination. The PCBM:MEH-PPV organic semiconductor absorbs light and the electrons are transferred to PCBM and holes remain in MEH-PPV and this generation gives photocarriers generated at organic-inorganic interface and in turn, the reverse current increases the efficiency substantially. The photovoltaic effect in $p$-Si/PCBM:MEH-PPV photodiode is based on the formation of excitons and subsequent dissociation and charge collection at the electrodes. The photocurrent is due to the carrier charges formed at the inorganic-organic interface as a result of electron-hole pair separation. The separated photocarriers are transported by the electrodes. The current at a given voltage for the $p$-Si/PCBM:MEH-PPV junction diode under illumination is higher than that of under dark. This indicates that the light illumination increases production of electron-hole pairs. The increase in charge production is dependent on the difference in the electron affinities between $p$-Si and PCBM:MEH-PPV semiconductors. The device shows a photovoltaic behavior with a maximum open circuit voltage $V_{\text {oc }}$ of $84 \mathrm{mV}$ and short-circuit current $I_{\mathrm{sc}}$ of $3.47 \mathrm{nA}$. The obtained values suggest that the $p$-Si/PCBM:MEH-PPV photovoltaic device can be operated as a heterojunction photodiode. Because solar cells are designed of course to minimize energy losses, the photodiodes are routinely designed to achieve a spectral response or a rapid time response [38].

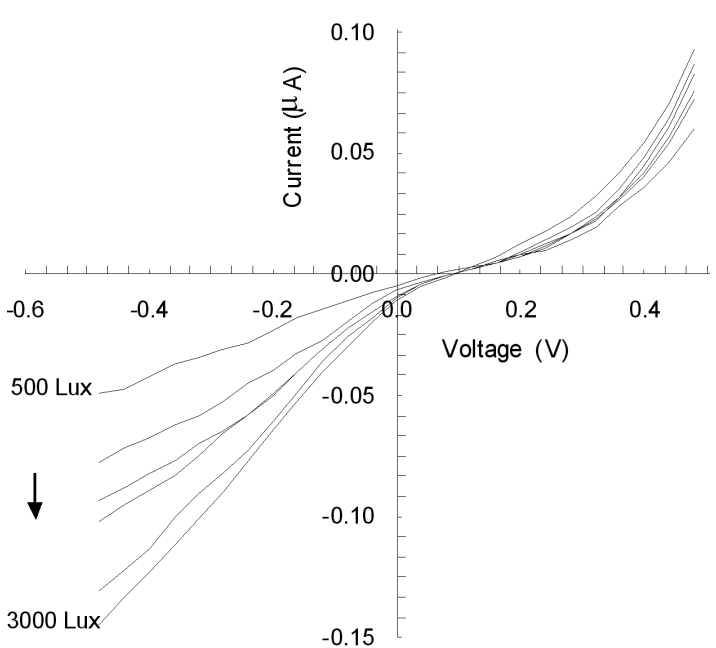

Fig. 7. $\quad I-V$ characteristics of the $p$-Si/PCBM:MEH-PPV diode at different illumination intensities.

\section{Conclusions}

Electronic properties of $p$-Si/PCBM:MEH-PPV $p-n$ junction diode have been investigated by current-voltage and capacitance-voltage methods. Consequently, $p$-Si/ PCBM:MEH-PPV device is a photodiode with the calculated electronic parameters, maximum open circuit voltage $V_{\mathrm{oc}}$ of $84 \mathrm{mV}$ and short-circuit current $I_{\mathrm{sc}}$ of $3.47 \mathrm{nA}$ under $6 \mathrm{~mW} / \mathrm{cm}^{2}$ illumination.

\section{Acknowledgments}

This work was supported by the Turkish Scientific and Technological Research Council of Turkey (TUBITAK) (project No. 105 T137) and also was partially supported by King Saud University under grant: KSU-VPP-102 and Feyzi AKKAYA Scientific Activates Supporting Fund (FABED).

\section{References}

[1] S. Gunster, S. Siebentritt, D. Meissner, Mol. Cryst. Liq. Cryst. 230, 351 (1993).

[2] K. Takahashi, S.I. Nakatani, T. Matsuda, H. Nambu, T. Kormura, K. Murata, Chem. Lett. 11, 2001 (1994).

[3] C.O. Too, G.G. Wallace, A.K. Burrell, G.E. Collis, D.L. Officer, E.W. Boge, S.G. Brodie, E.J. Evans, Synth. Met. 123, 53 (2001).

[4] K.M. Coakley, M.D. McGehee, Chem. Mater. 16, 4533 (2004)

[5] A. Goetzberger, C. Hebling, H.W. Schock, Mater. Sci. Eng. 40, 1 (2003). 
[6] J. Brabec, Sol. Energy Mater. Sol. Cells 83, 273 (2004).

[7] C.J. Brabec, N.S. Sariciftci, J.C. Hummelen, Adv. Funct. Mater. 11, 15 (2001).

[8] Organic Photovoltaics: Mechanism, Materials and Devices, Eds. S. Sun, N.S. Sariciftici, CRC Press, Boca Raton (FL) 2005.

[9] P. Peumans, A. Yakimov, S.R. Forrest, J. Appl. Phys. 93, 3693 (2003).

[10] H. Spanggaard, F.C. Krebs, Sol. Energy Mater. Sol. Cells 83, 125 (2004).

[11] T. Yohannes, T. Solomon, O. Inganäs, Synth. Met. 82, 215 (1996).

[12] L.S. Roman, M.R. Andersson, T. Yohannes, O. Inganäs, Adv. Mater. 9, 1164 (1997).

[13] L.S. Roman, W. Mammo, L.A.A. Pettersson, M.R. Andresson, O. Inganäs, Adv. Mater. 10, 774 (1998).

[14] D. Chirvase, Z. Chigurave, M. Knipper, J. Parisi, V. Dyakonov, J.C. Hümmelen, J. Appl. Phys. 93 , 3376 (2003)

[15] M. Çakar, Y. Onganer, A. Türüt, Synth. Met. 126 , 213 (2002).

[16] M.E. Aydın, T. Kılıçoğlu, K. Akkılıç, H. Hoşgören, Physica B 381, 113 (2006).

[17] K. Akkılıç, M.E. Aydın, İ. Uzun, T. Kılıçoğlu, Synth. Met. 156, 958 (2006).

[18] F. Yakuphanoglu, Sol. Energy Mater. Sol. Cells 91, $1182(2007)$

[19] K. Akkılıç, Ý. Uzun, T. Kılıçoğlu, Synth. Met. 157, 297 (2007).

[20] R. Pérez, N.J. Pinto, A.T. Johnson Jr., Synth. Met. 157, 231 (2007).

[21] F. Yakuphanoglu, Physica B: Condens. Matter 388, 226 (2007).

[22] M.M. El-Nahass, K.F. Abd-El-Rahman, A.A.M. Farag, A.A.A. Darwish, Organic Electron. 6, 129 (2005).
[23] F. Yakuphanoglu, Burm-Jong Lee, Physica B 390 , 151 (2007).

[24] F. Yakuphanoglu, Physica B 389, 306 (2007).

[25] I.J. Dittmer, K. Petrich, E.A. Marseglia, R.H. Friend, H. Rost, A.B. Holmes, Synth. Met. 102, 879 (1999).

[26] S.-S. Kim, J. Jo, C. Chun, J.-C. Hong, D.-Y. Kim, J. Photochem. Photobiol. A: Chem. 188, 364 (2007).

[27] A. Ltaief, J. Davenas, A. Bouazizi, R. Ben Chaabane, P. Alcouffe, H. Ben Ouada, Mater. Sci. Eng. C 25, 67 (2005).

[28] L.S. Roman, M.R. Andersson, T. Yohannes, O. Inganas, Adv. Mater. 9, 1164 (1997).

[29] S.E. Shaheen, C.J. Brabec, N.S. Sariciftci, Appl. Phys. Lett. 78, 841 (2001).

[30] E.H. Rhoderick, R.H. Williams, MetalSemiconductor Contacts, 2nd ed., Clarendon Press, Oxford 1988.

[31] S. Aydoğan, M. Sağlam, A. Türüt, Polymer 46, 10982 (2005).

[32] S.K. Cheung, N.W. Cheung, Appl. Phys. Lett. 49, 85 (1986).

[33] J.G. Simmons, Phys. Rev. Lett. 15, 967 (1965).

[34] S.R. Forrest, P.H. Schmidt, J. Appl. Phys. 59, 513 (1986).

[35] S.C. Jain, A.K. Kapoor, W. Geens, J. Poortmans, R. Mertens, M. Willander, J. Appl. Phys. 92, 3579 (2002).

[36] S.M. Sze, Physics of Semiconductor Devices, 2nd ed., Wiley, New York 1981.

[37] S. Aydoğan, M. Sağlam, A. Türüt, Polymer 46, 10982 (2005).

[38] R.F. Pierret, Semiconductor Device Fundamentals, Addison-Wesley Publ. Co., New York 1996. 